\title{
Histological features of medullary carcinoma and prognosis in triple-negative basal-like carcinomas of the breast
}

\author{
Felicia Marginean, Emad A Rakha, Bernard C Ho, Ian O Ellis and Andrew HS Lee \\ Department of Histopathology, Nottingham University Hospitals, Nottingham, UK
}

\begin{abstract}
Medullary carcinomas have a better prognosis than other grade 3 mammary carcinomas, but they typically show basal-like biological features, which are associated with a poor prognosis. In this study we examined the associations and prognostic relevance of medullary histological features in a series of 165 invasive carcinomas with a basal-like phenotype: triple-negative (oestrogen receptor, progesterone receptor, HER2) and expressing at least one basal marker (CK5/6, CK14, CK17 or EGFR). The following histological features were associated with each other: prominent inflammation, anastomosing sheets, absence of fibrosis, absence of infiltrative margin and absence of gland formation. Prominent inflammation and anastomosing sheets in at least $30 \%$ of the tumour were associated with a better prognosis on univariate analysis. The combination of these two features (a simplified definition of medullary-like type) was present in $17 \%$ of tumours and was an independent prognostic factor on multivariate analysis. This simplified definition had good inter-observer reproducibility $(\kappa=0.61)$ and is worthy of more detailed assessment in an unselected group of mammary carcinomas. A fibrotic focus was present in $36 \%$ of carcinomas. Only $3 \%$ of tumours with a fibrotic focus had features of medullary-like carcinomas. Fibrotic focus of greater than $30 \%$ of the tumour was associated with a poor prognosis. This study emphasizes the heterogeneity of morphology and behaviour of triple-negative basal-like carcinomas.
\end{abstract}

Modern Pathology (2010) 23, 1357-1363; doi:10.1038/modpathol.2010.123; published online 25 June 2010

Keywords: basal; human breast carcinoma; inflammation; medullary; prognosis; triple-negative

The recent global gene expression profiling studies of breast cancer have identified distinct molecular classes with clinical and biological relevance, namely luminal, HER2 and basal-like groups., ${ }^{1,2}$ The main immunophenotype of the basal-like class of tumours identified by these studies is the lack of hormone receptor expression and HER2 amplification (triple-negative phenotype: oestrogen receptor (ER), progesterone receptor (PR) and HER2) and the expression of basal-associated markers such as basal cytokeratins (CK5/6, CK17 and CK14) and EGFR. These are currently used as surrogate immunohistochemical markers for the identification of basal-like tumours in routine practice. ${ }^{3,4}$ Although triple-negative and basal-like phenotypes are not synonymous, ${ }^{5,6}$ they overlap $(60-90 \%$ of

Correspondence: Dr AHS Lee, FRCPath, Department of Histopathology, Nottingham University Hospitals, City Hospital Campus, Hucknall Road, Nottingham, NG5 1PB, UK.

E-mail: andrew.lee@nuh.nhs.uk

Received 31 December 2009; revised 11 May 2010; accepted 17 May 2010; published online 25 June 2010 triple-negative tumours are basal-like) and show common histological features. These include high histological grade (particularly high mitotic count), circumscribed edge, central fibrosis, necrosis, prominent lymphocytic infiltrate, syncytial growth and spindle cells. ${ }^{7,8}$

The basal-like class has a poor prognosis, but includes medullary carcinoma, ${ }^{9}$ which has a better prognosis than other grade 3 carcinomas. ${ }^{10}$ The Ridolfi criteria for the diagnosis of typical medullary carcinoma include more than $75 \%$ of the carcinoma composed of syncytial sheets, moderate or marked mononuclear infiltrate, pleomorphic nuclei, circumscribed edge, and absence of glandular differentiation and of ductal carcinoma in situ. However there is difficulty in reproducibly making this diagnosis. ${ }^{11-13}$ Also, if the Ridolfi criteria are strictly applied then less than $1 \%$ of carcinomas are of this type. $^{14}$

In addition, medullary carcinomas also share histological and immunophenotypic features with tumours that arise in carriers of BRCA1 germ-line mutations, which have a poor prognosis. ${ }^{15}$ 
This study aimed to test the hypotheses that medullary histological features are associated with each other and with a better prognosis in invasive basal-like carcinomas.

\section{Materials and methods}

This study was based on a well-characterized series of TNM stage T1 and T2 primary operable invasive breast carcinoma cases entered into the Nottingham Tenovus Primary Breast Carcinoma Series between 1989 and 1998. Patients were under the age of 71 and treated according to the local protocols. Patients' clinical history and tumour characteristics, information on therapy, tumour recurrence and survival were collected as previously described. ${ }^{5,8}$

Breast cancer tissue microarrays were prepared and immunohistochemically stained for ER, PR, HER2, CK5/6, CK14, CK17 and EGFR as previously described. ${ }^{8,16,17}$ Cut-off values for these biomarkers were the same as for previously reported patient series. Of the 1726 tumours informative for ER, PR and HER2, $282(16.3 \%)$ showed a triple-negative phenotype. Of these, 165 tumours expressing one or more of the basal markers (CK5/6, CK14, CK17 and/or EGFR) $)^{3,18-20}$ and with haematoxylin-andeosin-stained slides containing sufficient tumour for assessment were included in this study.

A total of 156 tumours were grade $3(95 \%)$ and 9 were grade 2 . The median size was $2.2 \mathrm{~cm}$. Axillary lymph nodes were positive in $54(33 \%)$ including 16 with three or more involved nodes. Seventeen tumours were categorized as medullary (typical or atypical) $)^{10}$ and 148 as of no special type including 1 tumour with myxoid stroma. Vascular invasion was present in $53(32 \%)$. The median patient age was 51 years. Seventy-one patients had a wide local excision and 94 received a mastectomy. Adjuvant chemotherapy was given to 70 patients and endocrine therapy to 31 (ER status was not used to select endocrine treatment at the start of the study period).

In this study, the following phenotypic features were assessed by two observers (FM and $\mathrm{AL}$ ) on a multi-headed microscope (see Figure 1). The intensity of lymphohistiocytic inflammation was assessed on a semi-quantitative scale as absent/ minimal, mild, moderate or marked, similar to the method described previously for assessing diffuse inflammation. ${ }^{14}$ Prominent inflammation was defined as moderate or marked inflammation. The proportion of the tumour showing the following
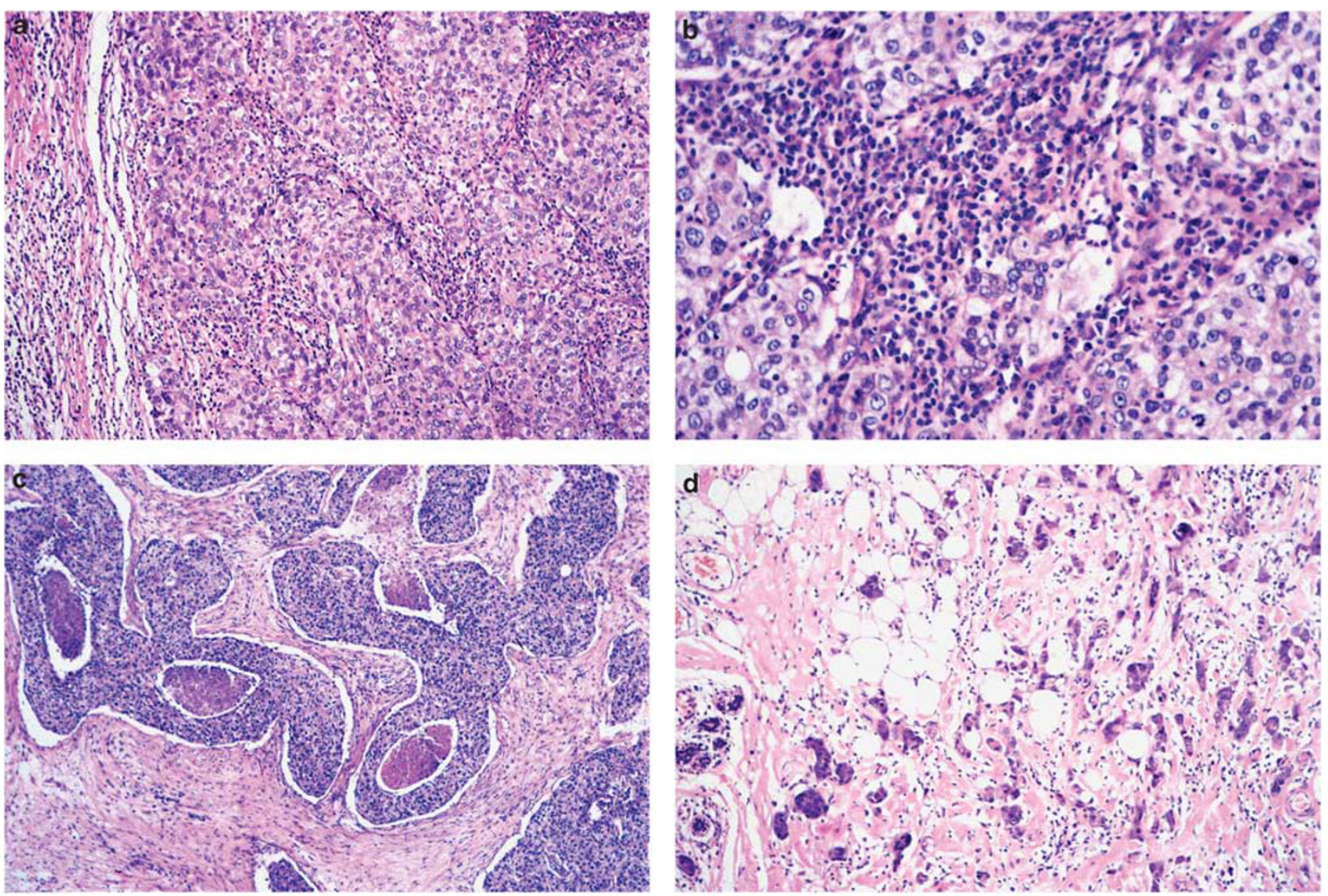

Figure 1 (a) Carcinoma with largely circumscribed edge, composed of anastomosing sheets of cells with no fibrosis and (b) with moderate inflammatory infiltrate. (c) Carcinoma composed of anastomosing sheets of cells with necrosis and prominent fibrosis. (d) Carcinoma with infiltrative margin and mild inflammation. 
features was estimated: anastomosing sheets (defined as interconnecting cohesive sheets of tumour cells visible at low power), fibrosis, infiltrative margin and gland formation. The proportion of the invasive carcinoma with necrosis was categorized as none, $1-10 \%$ and more than $10 \%$. The mitotic score and nuclear pleomorphism score components of histological grade were obtained from the pathology reports. ${ }^{21}$ The presence or absence of ductal carcinoma in situ was noted. Two observers (ER and AL) assessed the proportion of tumour showing features of a fibrotic focus using the definition of Copaert on a multi-headed microscope. Fibrotic focus was defined by Hasebe et $a l^{22}$ as a central area of fibrosis containing fibroblasts and/or collagen. Necrosis and elastosis may also be present. Colpaert et $a l^{23}$ refined this definition by adding that there must be an area of fibrosis at least $3 \mathrm{~mm}$ across with no carcinoma cells. To measure inter-observer variation, a third observer assessed inflammation and anastomosing sheets as defined above in 52 tumours.

\section{Statistical Analysis}

The relationship between different histological features was analysed with the Spearman's rank correlation coefficient. In view of the multiple comparisons only results with $P<0.005$ were regarded as significant.

Survival analysis was based on breast cancer deaths, using the time from the initial surgery to death from breast cancer. Deaths due to other causes were censored. Survival curves were analysed by the Kaplan-Meier method and significance was determined by the log-rank test. ${ }^{24}$ Multivariate analysis was performed by Cox proportional hazards analysis. A $P$-value $<0.05$ (two sided) was considered statistically significant. Follow-up was avail- able for 158 patients. The median follow-up was 9.2 years (range, 0.3-20.2) with 60 breast cancer deaths.

This study was approved by Nottingham Research Ethics Committee 2 under the title of 'Development of a molecular genetic classification of breast cancer'.

\section{Results}

The following histological features were associated with each other: prominent inflammation, anastomosing sheets, absence of fibrosis, absence of infiltrative margin and absence of gland formation (Tables 1 and 2). The percentage of fibrotic focus correlated with the percentage of fibrosis, necrosis and absence of anastomosing sheets.

On univariate analysis, breast-cancer-specific survival was better in patients with tumours showing moderate or marked inflammation than in patients with tumours showing absent or mild inflammation (Figure 2a). The survival curves for absent and mild inflammation were very similar (data not shown). Prominent inflammation (moderate or marked) was present in $31 \%$ of tumours.

Table 2 The relationship between the proportion of the tumour composed of anastomosing sheets and the intensity of inflammation

\begin{tabular}{lrrrr}
\hline Inflammation & \multicolumn{3}{c}{ Anastomosing sheets } & \multirow{2}{*}{ Total } \\
\cline { 2 - 4 } & $0-29 \%$ & $30-69 \%$ & $70-100 \%$ & \\
\hline Absent & 19 & 2 & 2 & 23 \\
Mild & 77 & 12 & 4 & 93 \\
Moderate & 19 & 12 & 11 & 42 \\
Marked & 2 & 3 & 2 & 7 \\
Total & 117 & 29 & 19 & 165 \\
& & & & \\
& & & &
\end{tabular}

Table 1 Correlation between different medullary-like histological features

\begin{tabular}{|c|c|c|c|c|c|c|c|c|c|}
\hline & $\begin{array}{c}\text { Anastomosing } \\
\text { sheets }\end{array}$ & Fibrosis & Necrosis & $\begin{array}{l}\text { Infiltrative } \\
\text { margin }\end{array}$ & Glands & DCIS & $\begin{array}{l}\text { Mitotic } \\
\text { score }\end{array}$ & $\begin{array}{c}\text { Nuclear } \\
\text { pleomorphism }\end{array}$ & Fibrotic focus \\
\hline Inflammation score & $\begin{array}{c}r_{s}=0.31 \\
P<0.0001\end{array}$ & $\begin{array}{l}r_{\mathrm{s}}=-0.40 \\
P<0.0001\end{array}$ & $\begin{array}{l}r_{\mathrm{s}}=0.10 \\
P=0.20\end{array}$ & $\begin{aligned} r_{\mathbf{s}} & =-0.23 \\
P & =0.003\end{aligned}$ & $\begin{array}{l}r_{\mathrm{s}}=-0.32 \\
P<0.0001\end{array}$ & $\begin{aligned} r_{\mathrm{s}} & =-0.16 \\
P & =0.04\end{aligned}$ & $\begin{aligned} r_{\mathrm{s}} & =-0.17 \\
P & =0.03\end{aligned}$ & $\begin{array}{l}r_{\mathrm{s}}=0.02 \\
P=0.79\end{array}$ & $\begin{array}{c}r_{\mathrm{s}}=-0.18 \\
P=0.02\end{array}$ \\
\hline Anastomosing sheets & & $\begin{array}{l}r_{\mathrm{s}}=-0.38 \\
P<0.0001\end{array}$ & $\begin{array}{l}r_{\mathrm{s}}=0.20 \\
P=0.01\end{array}$ & $\begin{array}{l}r_{\mathrm{s}}=-0.62 \\
P<0.0001\end{array}$ & $\begin{array}{l}r_{\mathrm{s}}=-0.33 \\
P<0.0001\end{array}$ & $\begin{array}{l}r_{\mathrm{s}}=-0.26 \\
P=0.001\end{array}$ & $\begin{array}{l}r_{\mathrm{s}}=0.17 \\
P=0.03\end{array}$ & $\begin{aligned} r_{\mathrm{s}} & =-0.04 \\
P & =0.57\end{aligned}$ & $\begin{aligned} r_{\mathrm{s}} & =-0.22 \\
P & =0.005\end{aligned}$ \\
\hline Fibrosis & & & $\begin{array}{c}r_{\mathrm{s}}=0.004 \\
P=0.95\end{array}$ & $\begin{array}{c}r_{\mathrm{s}}=0.34 \\
P<0.0001\end{array}$ & $\begin{array}{c}r_{\mathrm{s}}=0.27 \\
P=0.0006\end{array}$ & $\begin{array}{c}r_{s}=0.36 \\
P<0.0001\end{array}$ & $\begin{aligned} r_{\mathrm{s}} & =-0.17 \\
P & =0.03\end{aligned}$ & $\begin{array}{l}r_{\mathrm{s}}=0.04 \\
P=0.64\end{array}$ & $\begin{array}{c}r_{\mathrm{s}}=0.36 \\
P<0.0001\end{array}$ \\
\hline Necrosis & & & & $\begin{array}{c}r_{\mathrm{s}}=0.27 \\
P=0.0004\end{array}$ & $\begin{array}{c}r_{\mathrm{s}}=-0.08 \\
P=0.27\end{array}$ & $\begin{aligned} \boldsymbol{r}_{\mathbf{s}} & =0.10 \\
P & =0.19\end{aligned}$ & $\begin{array}{c}r_{\mathrm{s}}=0.22 \\
P=0.006\end{array}$ & $\begin{array}{l}r_{\mathrm{s}}=0.15 \\
P=0.05\end{array}$ & $\begin{array}{c}r_{\mathrm{s}}=0.30 \\
\boldsymbol{P}=\mathbf{0 . 0 0 0 1}\end{array}$ \\
\hline Infiltrative margin & & & & & $\begin{array}{c}r_{\mathrm{s}}=0.32 \\
P<0.0001\end{array}$ & $\begin{array}{c}r_{\mathrm{s}}=0.23 \\
P=\mathbf{0 . 0 0 3}\end{array}$ & $\begin{aligned} r_{\mathbf{s}} & =-0.25 \\
P & =\mathbf{0 . 0 0 1}\end{aligned}$ & $\begin{array}{l}r_{\mathrm{s}}=0.05 \\
P=0.50\end{array}$ & $\begin{array}{l}r_{\mathrm{s}}=0.09 \\
P=0.23\end{array}$ \\
\hline Gland formation & & & & & & $\begin{array}{c}r_{\mathrm{s}}=0.23 \\
P=0.004\end{array}$ & $\begin{array}{c}r_{\mathrm{s}}=-0.24 \\
P=0.002\end{array}$ & $\begin{array}{l}r_{\mathrm{s}}=0.06 \\
P=0.43\end{array}$ & $\begin{array}{l}r_{\mathrm{s}}=0.07 \\
P=0.39\end{array}$ \\
\hline DCIS & & & & & & & $\begin{array}{c}r_{\mathrm{s}}=-0.17 \\
P=0.03\end{array}$ & $\begin{array}{l}r_{\mathrm{s}}=0.13 \\
P=0.66\end{array}$ & $\begin{array}{l}r_{\mathrm{s}}=0.18 \\
P=0.02\end{array}$ \\
\hline Mitotic score & & & & & & & & $\begin{array}{l}r_{\mathrm{s}}=0.09 \\
P=0.66\end{array}$ & $\begin{array}{c}r_{\mathrm{s}}=0.006 \\
P=0.94\end{array}$ \\
\hline Nuclear pleomorphism & & & & & & & & & $\begin{array}{c}r_{\mathrm{s}}=-0.03 \\
P=0.66\end{array}$ \\
\hline
\end{tabular}

Results with $P<0.005$ are marked in bold. 

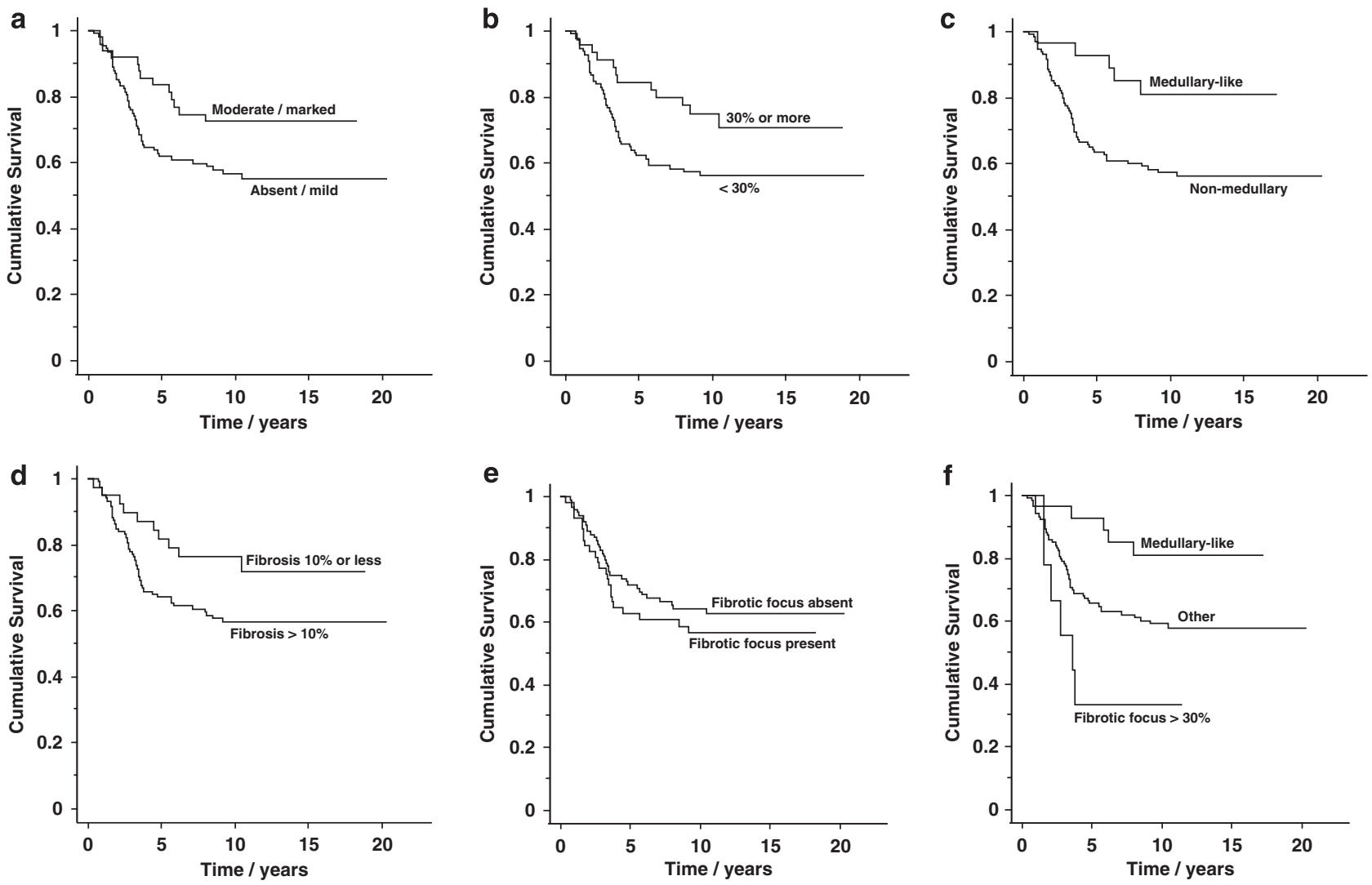

Figure 2 Breast-cancer-specific survival was associated with (a) intensity of inflammation $(P=0.04)$, (b) anastomosing sheets $(P=0.04)$, (c) the combination of prominent inflammation and anastomosing sheets (medullary-like) compared with one or neither of these features $(P=0.02)$ and $(\mathbf{d})$ fibrosis $(P=0.06)$. Breast-cancer-specific survival was $(\mathbf{e})$ not associated with the presence of a fibrotic focus $(P=0.36)$, (f) but was associated with a fibrotic focus greater than $30 \%$ compared with medullary-like and other carcinomas.

In initial analysis of anastomosing sheets, the tumours were divided into three groups: $<30$, 30-69 and $70 \%$ or more. The latter two groups had similar survival, so they were combined (29\% of tumours). Tumours composed of at least $30 \%$ anastomosing sheets were associated with a better survival (Figure 2b).

The combination of prominent inflammation and anastomosing sheets in at least $30 \%$ of the tumour ('medullary-like', $17 \%$ of tumours) produced even clearer separation of the survival curves (Figure 2c). There was an overlap between medullary-like carcinomas as defined above and medullary carcinomas using conventional criteria $\left(\chi^{2}=14.7, P=0.0001\right)$, but some atypical medullary carcinomas did not fall within the medullary-like group. DCIS was seen less often in medullary-like carcinomas compared with other carcinomas (32 vs $64 \%, \chi^{2}=8.2, P=0.004$ ). The presence of DCIS was not associated with prognosis in the whole series or in medullary-like carcinomas. Medullary-like carcinomas were more often node negative $(P=0.006)$, but survival analysis in node-negative carcinomas showed that medullary-like type was still associated with a better prognosis $(P=0.025)$. Histological grade, tumour size, proportion of tumours showing necrosis
( $>10 \%$ necrosis in $39 \%$ of both categories) and proportion of patients receiving chemotherapy were similar in medullary-like and other carcinomas.

In initial analysis of fibrosis the tumours were divided into three groups: $10 \%$ or less, $11-29$ and $30 \%$ or more. The latter two groups had similar survival, so they were combined. Fibrosis of $10 \%$ or less of the tumour ( $25 \%$ of tumours) was associated with a better survival, but this was of borderline significance (Figure 2d).

A fibrotic focus was present in 60 carcinomas $(36 \%)$. Only two tumours with a fibrotic focus showed medullary-like features. The fibrotic focus in these two tumours affected 10 and $20 \%$ of the tumours. There was no difference in nodal stage, histological grade, tumour size or vascular invasion between tumours with or without a fibrotic focus. Patients with tumour containing a fibrotic focus received chemotherapy more often than patients with a tumour without a fibrotic focus (57 vs 39\%, $P=0.05$ ). When considering all tumours with a fibrotic focus, there was no association between this feature and survival (Figure 2e). However, a fibrotic focus that replaced more than $30 \%$ of the tumour (similar to the cut-off of greater than a third used by Colpaert et $\mathrm{al}^{23}$ ) was associated with a worse 
prognosis $(P=0.03)$, but only 11 tumours satisfied this criterion. When tumours were divided into medullary-like, fibrotic focus larger than $30 \%$ and tumours not falling into either of these groups there was clear separation of the survival curves (Figure 2f). None of the other histological features assessed was associated with survival.

On univariate analysis, breast-cancer-specific survival was associated with nodal status, tumour size and vascular invasion, but not with histological grade ( $95 \%$ of tumours were grade 3 ), patient age, chemotherapy or endocrine therapy. On multivariate analysis including medullary-like type and traditional factors, the only factors associated with survival were medullary-like type $(P=0.02$, relative risk 0.32 (95\% CI $0.13-0.81)$ ) and tumour size $(P=0.02$, relative risk 1.4 (95\% CI $1.06-2.0))$. On multivariate analysis including fibrotic focus greater than $30 \%$ and traditional factors, the only factors associated with survival were fibrotic focus $(P=0.02$, relative risk 3.3 (95\% CI $1.2-8.5))$ and lymph node stage $(P=0.02$, relative risk 1.7 (95\% CI 1.08-2.5)). Similarly, tumour type, divided into medullary-like, fibrotic focus greater than $30 \%$ and other, was an independent prognostic factor in addition to lymph node stage.

The study of reproducibility found 90\% agreement in assessing the combination of prominent inflammation and anastomosing sheets $(\kappa=0.61)$.

\section{Discussion}

This study assessed medullary histological features in a well-defined group of carcinomas that showed basal-like phenotype defined as being triple-negative with expression of at least one of the basal markers used. ${ }^{3,5}$ The major findings were an association of medullary histological features with each other and association of prominent inflammation and anastomosing sheets with better prognosis. In addition, a separate group with a large fibrotic focus associated with a poor prognosis was identified.

Prominent inflammation was present in $31 \%$ of tumours compared with $10 \%$ in unselected carcinomas. ${ }^{10,14}$ This is, at least in part, likely to be linked to histological grade as there is strong correlation between histological grade and intensity of inflammation ${ }^{10}$ and $95 \%$ of tumours in the present series were grade 3 . The separation of inflammation into two groups (absent and mild vs moderate and marked) was based on previous studies. ${ }^{10,14,25}$ The finding in this study that absent and mild inflammation were associated with a similar prognosis and that both had a worse prognosis than moderate and marked inflammation supports this cut-off. The finding of an association of prominent inflammation with better prognosis has been described in triplenegative carcinomas ${ }^{26}$ and is similar to the majority of studies of unselected carcinomas. ${ }^{10,14}$
We chose to use the feature of anastomosing sheets rather than syncytial sheets as proposed by Ridolfi. Classification as a syncytial sheet requires recognition of indistinct cell borders at high magnification (which we found very subjective) in addition to the recognition of the anastomosing sheets. We found that assessment of anastomosing sheets could be carried out easily at low magnification. These two features are often present. Ridolfi suggested syncytial sheets should be present in at least $75 \%$ of the tumour in a typical medullary carcinoma. In this study, tumours composed of at least $30 \%$ anastomosing sheets were associated with a better survival. There was no difference in survival for patients with tumours with 30-69\% anastomosing sheets and those with tumours with at least $70 \%$ anastomosing sheets.

Fibrotic focus is a recently described histological feature associated with high histological grade, larger tumour size, vascular invasion, nodal metastasis, tumour necrosis, HER2 expression and poor prognosis. $^{22,23}$ Central fibrosis is more frequently seen in basal-like carcinomas identified with immunohistochemistry or gene expression profiles. ${ }^{7,27}$ Central fibrosis was found in $51 \%$ of tumours and associated with worse prognosis in a study of 97 triple-negative carcinomas. ${ }^{26}$ In this study a fibrotic focus was present in $36 \%$ of tumours and there was almost no overlap with medullary-like features. Fibrotic focus was only of prognostic significance when the feature was extensive (more than $30 \%$ of the tumour area). We chose to use the Colpaert et al definition of fibrotic focus, which requires a central area of fibrosis without carcinoma at least $3 \mathrm{~mm}$ across, as this is more objective than Hasebe's definition stating that 'tumor cells are seldom seen in fibrotic focus $\leq 3 \mathrm{~mm}$ in size'. A large fibrotic focus is easy to recognize. However, some basal-like carcinomas show prominent central fibrosis, but with scattered foci of carcinoma throughout, which do not fit the definition by Colpaert et al.

We found assessment of the tumour margin difficult. It is easy to recognize a tumour that is clearly infiltrative, but much more subjective to say if it is circumscribed. Others have found poor reproducibility in assessing circumscription of margins compared with assessment of syncytial growth pattern and intensity of mononuclear infiltrate. ${ }^{28}$

The association of medullary features with each other in the present series supports the concept of a medullary histological type. Previous studies have found that medullary carcinomas have a better prognosis than other grade 3 carcinomas. ${ }^{10}$ This study of basal-like carcinomas found a better prognosis associated with a less strict definition of medullary-like carcinoma (prominent inflammation and at least $30 \%$ anastomosing sheets).

The Ridolfi criteria are very restrictive and may therefore miss cases with a good prognosis: in a recent study using the criteria less than $1 \%$ of 
carcinomas were classified as medullary. ${ }^{14}$ Pedersen et $a l^{29}$ proposed a simplified definition of medullary carcinoma: syncytial growth pattern in more than $75 \%$ of the tumour and diffuse moderate or marked mononuclear infiltration, which was reproducible and prognostically significant. The data in this study suggest modifying the Pedersen definition by reducing the proportion of the tumour showing anastomosing sheets required for the diagnosis. This study only examined a small subset of carcinomas and it would be useful to investigate the revised criteria in a large series of unselected mammary carcinomas. A further value of a less strict definition is that it would help in the recognition of BRCA-1mutation-associated carcinoma, which frequently shows medullary-like features in addition to the other characteristic clinical and immunophenotypic features.

In this study there was substantial inter-observer agreement $(\kappa=0.61)$ using the simplified definition of medullary-like carcinoma. This compares favourably with previous studies of the Ridolfi criteria that have shown $\kappa$ values from 0.10 to $0.55 .{ }^{12,28} \mathrm{In}$ keeping with this, Gaffey et $a l^{11}$ found that the Pedersen definition, which is similar to the proposed definition, was more reproducible than the Ridolfi definition.

The findings of this study also emphasize the heterogeneity of morphology and behaviour of basallike carcinomas and confirm the results of previous studies that indicate that a subgroup of basallike tumours is associated with better prognosis (reviewed in Rakha et $a l^{4}$ ).

This study has found that medullary histological features correlate with each other in triple-negative basal-like carcinomas. A simplified definition of medullary-like carcinoma was associated with better prognosis in this group of carcinomas. Inter-observer reproducibility for this definition was good. Further investigation of this definition in unselected mammary carcinomas would be useful.

\section{Disclosure/conflict of interest}

The authors declare no conflict of interest.

\section{References}

1 Perou CM, Sorlie T, Eisen MB, et al. Molecular portraits of human breast tumours. Nature 2000;406: 747-752.

2 Sorlie T, Tibshirani R, Parker J, et al. Repeated observation of breast tumor subtypes in independent gene expression data sets. Proc Natl Acad Sci USA 2003;100:8418-8423.

3 Cheang MC, Voduc D, Bajdik C, et al. Basal-like breast cancer defined by five biomarkers has superior prognostic value than triple-negative phenotype. Clin Cancer Res 2008;14:1368-1376.
4 Rakha EA, Reis-Filho JS, Ellis IO. Basal-like breast cancer: a critical review. J Clin Oncol 2008;26:2568-2581.

5 Rakha EA, Elsheikh SE, Aleskandarany MA, et al. Triple-negative breast cancer: distinguishing between basal and nonbasal subtypes. Clin Cancer Res 2009;15: 2302-2310.

6 Rakha E, Ellis I, Reis-Filho J. Are triple-negative and basal-like breast cancer synonymous? Clin Cancer Res 2008;14:618; author reply 618-619.

7 Fulford LG, Easton DF, Reis-Filho JS, et al. Specific morphological features predictive for the basal phenotype in grade 3 invasive ductal carcinoma of breast. Histopathology 2006;49:22-34.

8 Rakha EA, Putti TC, Abd El-Rehim DM, et al. Morphological and immunophenotypic analysis of breast carcinomas with basal and myoepithelial differentiation. J Pathol 2006;208:495-506.

9 Jacquemier J, Padovani L, Rabayrol L, et al. Typical medullary breast carcinomas have a basal/myoepithelial phenotype. J Pathol 2005;207:260-268.

10 Rakha EA, Aleskandarany M, El-Sayed ME, et al. The prognostic significance of inflammation and medullary histological type in invasive carcinoma of the breast. Eur J Cancer 2009;45:1780-1787.

11 Gaffey MJ, Mills SE, Frierson HF, et al. Medullary carcinoma of the breast: interobserver variability in histopathologic diagnosis. Mod Pathol 1995;8:31-38.

12 Anderson TJ, Sufi F, Ellis IO, et al. Implications of pathologist concordance for breast cancer assessments in mammography screening from age 40 years. Hum Pathol 2002;33:365-371.

13 Pedersen L, Holck S, Schiodt T, et al. Inter- and intraobserver variability in the histopathological diagnosis of medullary carcinoma of the breast, and its prognostic implications. Breast Cancer Res Treat 1989;14:91-99.

14 Lee AHS, Gillett CE, Ryder K, et al. Different patterns of inflammation and prognosis in invasive carcinoma of the breast. Histopathology 2006;48:692-701.

15 Stoppa-Lyonnet D, Ansquer Y, Dreyfus H, et al. Familial invasive breast cancers: worse outcome related to BRCA1 mutations. J Clin Oncol 2000;18:4053-4059.

16 Abd El-Rehim DM, Ball G, Pinder SE, et al. Highthroughput protein expression analysis using tissue microarray technology of a large well-characterised series identifies biologically distinct classes of breast cancer confirming recent cDNA expression analyses. Int J Cancer 2005;116:340-350.

17 Rakha EA, El-Sayed ME, Green AR, et al. Prognostic markers in triple-negative breast cancer. Cancer 2007;109:25-32.

18 Nielsen TO, Hsu FD, Jensen $\mathrm{K}$, et al. Immunohistochemical and clinical characterization of the basal-like subtype of invasive breast carcinoma. Clin Cancer Res 2004;10:5367-5374.

19 Rakha EA, El-Sayed ME, Green AR, et al. Breast carcinoma with basal differentiation: a proposal for pathology definition based on basal cytokeratin expression. Histopathology 2007;50:434-438.

20 Sasa M, Bando Y, Takahashi M, et al. Screening for basal marker expression is necessary for decision of therapeutic strategy for triple-negative breast cancer. J Surg Oncol 2008;97:30-34.

21 Elston CW, Ellis IO. Pathological prognostic factors in breast cancer I. The value of histological grade in breast cancer: experience from a large study with longterm follow-up. Histopathology 1991;19:403-410. 
22 Hasebe T, Sasaki S, Imoto S, et al. Prognostic significance of fibrotic focus in invasive ductal carcinoma of the breast: a prospective observational study. Mod Pathol 2002;15:502-516.

23 Colpaert C, Vermeulen P, van Beest P, et al. Intratumoral hypoxia resulting in the presence of a fibrotic focus is an independent predictor of early distant relapse in lymph node-negative breast cancer patients. Histopathology 2001;39:416-425.

24 Kaplan EL, Meier P. Non-parametric estimation from incomplete observations. J Am Stat Assoc 1958;53: 457-481.

25 Rilke F, Colnaghi MI, Cascinelli N, et al. Prognostic significance of HER-2/neu expression in breast cancer and its relationship to other prognostic factors. Int J Cancer 1991;49:44-49.

26 Kreike B, van Kouwenhove M, Horlings H, et al. Gene expression profiling and histopathological character- ization of triple-negative/basal-like breast carcinomas. Breast Cancer Res 2007;9:R65.

27 Van den Eynden GG, Smid M, Van Laere SJ, et al. Gene expression profiles associated with the presence of a fibrotic focus and the growth pattern in lymph nodenegative breast cancer. Clin Cancer Res 2008;15: 2944-2952.

28 Rigaud C, Theobald S, Noel P, et al. Medullary carcinoma of the breast. A multicenter study of its diagnostic consistency. Arch Pathol Lab Med 1993; 117:1005-1008.

29 Pedersen L, Zedeler K, Holck S, et al. Medullary carcinoma of the breast, proposal for a new simplified histopathological definition. Based on prognostic observations and observations on inter- and intraobserver variability of 11 histopathological characteristics in 131 breast carcinomas with medullary features. Br J Cancer 1991;63:591-595. 